\title{
Largely Dismissed Heart Failure Drug May Help Solve Costly Problem for Medicare and Hospitals
}

\section{William H Wehrmacher*}

Clinical Professor of Medicine and Adjunct Professor of Physiology, Loyola University of Chicago Medical School in Maywood, Illinois, USA

Targeted for reduction, health care reform is targeting on Medicare expenses resulting from readmission of hospitalized Medicare beneficiaries within 30-days for all-causes. Annually this costs 17 billion dollars. Heart failure is a leading cause of both hospital admission and readmission for older adults. Digitalis therapy has been neglected for treating heart failure among such adults although it is effective and inexpensive and shows great promise for reducing need for both hospital admission and readmission [1].

This neglect has occurred since the DIG report [2] in 1994 showed no reduction in morality but confirmed effectiveness of digoxin (one of the pure glycosides of digitalis) to reduce symptoms and the need for hospitalization. In spite of these clear benefits, the troublesomeness of frequent dosage adjustments, discouraged use. Considerable skill was required to capture the window of therapeutic effectiveness, between serious toxicity and safe adequate effective therapy [3]. Digitalis rapidly disappeared from general use.

In 1996, the benefits of digitalis were rediscovered [4] but its use did not increase. In 2007, evidence was published [5] to show that it also increased survival. None of this evidence restored faith in or restored utilization of digitalis for therapy.

This year, Ali Ahmed, professor of geriatrics at the University of
Alabama in Birmington along with coworkers from other leading medical centers [1] presented evidence at the March Annual Scientific Session of the American College of Cardiology that "Digoxin reduces 30-day all-cause hospital admission in ambulatory older patients with chronic systolic heart failure." They are in the process of developing future studies need to examine its effect on 30-day all-cause hospital readmission in hospitalized patients with acute heart failure.

Isn't time to provide digitalis for heart failure and to provide the savings that it offers for the costs of medical care?

\section{References}

1. Ahmed Ali, et al. (2013) Largely dismissed heart failure drug may help solve costly problem for Medicare and hospitals. The American Journal of Medicine.

2. (1997) "The Effect of Digoxin on Mortality and morbidity in Patients with heart Failure". The Digitalis Investigation Group. N Engl J Med 336: 525-533.

3. Wehrmacher WH (in press) "Illuminating the Third Millennium with Flashes of experience from the $20^{\text {th }}$ Century" Hektoen International in press.

4. Wehrmacher WH (1996) "Digitalis Rediscovered: 1996 Results of the DIG Trial in Perspective". Internal Medicine World Report Cardiology 36.

5. Wehrmacher WH (2007) Digitalis Treatment Decreases Mortality and Morbidity in Heart Failure Patients. Reanalysis of Digitalis Treatment. Cardiology 108: 157-158.
*Corresponding author: William H Wehrmacher, M.D.,F A.C.C., F.A.C.P., Clinical Professor of Medicine and Adjunct Professor of Physiology, Loyola University of Chicago Medical School in Maywood, Illinois, USA, E-mail: wwehrma@lumc.edu

Received April 16, 2013; Accepted April 18, 2013; Published April 20, 2013

Citation: Wehrmacher WH (2013) Largely Dismissed Heart Failure Drug May Help Solve Costly Problem for Medicare and Hospitals. J Vasc Med Surg 1: e102. doi:10.4172/2329-6925.1000e102

Copyright: @ 2013 Wehrmacher WH. This is an open-access article distributed under the terms of the Creative Commons Attribution License, which permits unrestricted use, distribution, and reproduction in any medium, provided the original author and source are credited. 